is that language matters: in effect, there is no such thing as 'casual' or 'low-level' abuse. And, as the ongoing comments from both men and women on social media make clear, the impact of such behaviour on women, many of whom are early in their careers, can be pernicious and long-lasting. Women can begin to doubt their achievements and their abilities. They might question the motives of people who comment on their work. In short, they can lose confidence; when combined with the structural and institutional obstacles that they already face, this can make women look elsewhere for job satisfaction. This is unacceptable. Science simply cannot afford to lose some of its best talent to boorishness.

A major problem is the widespread tacit acceptance of adolescent behaviour. Let us call him Dr Inappropriate: he is the lecturer at the conference drinks reception with the wandering hands. (No such behaviour has been attributed to Zivkovic.) He is the head of department who thanks his female colleague for her excellent presentation but suggests that she wears a shorter skirt next time (yes, this really happened). Worse, Dr Inappropriate is often the lab head, or an equivalent - a mentor with responsibility and power over the careers of the women whom he asks to work late on a project or to join him in a taxi home. Sometimes he is a very senior scientist indeed.

Nature acknowledged in an Editorial last year that we have poor representation of women among reviewers and authors (see Nature 491,$495 ; 2012$ ) - but we pledged to change and have attempted to do so, with mixed results that we shall report soon. We have asked others to acknowledge their own gender biases, and urged them to do what they can to improve the prospects and visibility of women in science.

Our Women in Science special issue this year (see nature.com/ women) offered our most comprehensive and high-profile collection of articles on the subject so far. Yet we have not adequately addressed the problem of harassment, perhaps because it is difficult to quantify. Officially, the obstacles to women in science are policy issues such as availability of childcare and lack of flexible hours. We might never know how many are pushed to leave because they are fed up of working with Dr Inappropriate. Just as worrying are those women who do not make that choice and who find that they must simply endure.

The evidence of the scale and depth of the problem is anecdotal. But the anecdotes all point to sexual harassment being a real stain on science. Just ask around: everyone knows a Dr Inappropriate. (We have here emphasized male-female harassment, but female-male and same-sex harassment happens too.)

"Science simply cannot afford to lose some of its best talent to boorishness."

What is to be done? Most institutions already have policies that outlaw harassment and bullying. Could and should they be more strictly enforced? Yes. This often requires the victim to make an official complaint, and many are justifiably reluctant to do so, but a facility for anonymous whistle-blowing may help. A more pragmatic solution is to force Dr Inappropriate to keep his hands to himself, and this is where the rest of us can come in. More of us must challenge such actions when we see them, publicly if necessary. Too often we accommodate and excuse them: "He doesn't mean it"; "That's what he's like after a drink"; "Just make sure you don't work late on your own."

There are many behaviours that could be construed as abuse, and there are grey zones. Flirting is human nature. Some students marry their supervisors. Such considerations argue against glib judgements, but must not distract from the central message.

Here is one category of sexual harassment to focus on: when it represents an abuse of a professional relationship, particularly one in which the abuser has power and the victim feels unable to challenge it as they would like. That is wrong, and we should all label it so. We should all seek to promote not only appropriate rules, but also a culture of active discouragement and prevention of sexual harassment. If you are the party with the power, ask yourself: will the recipient of your social overtures wonder whether your support for his or her work is dependent on how she or he responds? If the answer is yes - or even maybe - do not cross that line.

\section{Magnetic map}

\section{Chemists present a way to infer the enigmatic temperature variations inside a reactor.}

$\mathrm{M}$ ost chemical products start their lives as oil. And most of the conversion processes used to turn the black stuff into plastics, fuels and the rest rely on catalysts. Given the sensitivity of catalysts and Earth's dwindling supplies of oil, you might think that these reactions would be among the most studied and the best understood in the chemist's cookbook.

Unfortunately not. In fact, for many chemists and chemical engineers - those who work with bucketloads of reactants rather than the contents of pipettes - what goes on inside an industrial reactor is something of a mystery. It's a black box. Indeed, when some textbooks and academic papers on the subject show flow charts of chemical processes, they actually represent the reactor, the beating heart of our industrial society, as a black box. If process engineers want to know what happens inside - and so how to make it more efficient, safer or more environmentally friendly - they measure what comes out, compare it with what goes in, and make an educated guess.

As computing power has grown, this educated guesswork has been renamed 'modelling'. Reconstructions of the catalytic processes that occur in reactors use complex mathematics to represent the relationship between reactants, products and everything in between. Heat transfer, fluid dynamics and surface-reaction kinetics all offer a theoretical platform for such models, but, like all models, they rely on observations from the real world to make them realistic. Which takes us back to the black box and, often, to the most basic of questions just how hot is it in there?

Anyone who has cooked a soufflé will know that the temperature, and how it fluctuates inside the oven, has a crucial bearing on the result. They know that the temperature selected and that the oven reaches can disagree. And they know that, even with the best temperature circulation, cool spots can lurk between lower shelves or above a baking tray. Now imagine that your precious pudding relies on the random collisions of a fizzing tempest of high-pressure gas and ageing, unpredictable catalysts. And that you are being asked to deliver 3,000 puddings an hour.

A reliable temperature map of the guts of a working chemical reactor would be valuable. People have tried to achieve this, most often by placing sensors at strategic points. The problem is the age-old paradox that the measurement disturbs what is being measured.

On page 537 of this issue, chemists offer a solution. Nanette Jarenwattananon at the University of California, Los Angeles, and her colleagues describe how they use the magnetic field of an nuclear magnetic resonance (NMR) scanner to accurately infer the hot and cold spots of a reactor carrying out the hydrogenation of propylene. And they report that, under the right conditions, hotter parts of the reactor signal narrower peaks on the NMR spectra.

There is a pleasing symmetry here. In the 1970s, NMR was handed to biologists and renamed magnetic resonance imaging (MRI). The biologists worked out a way to use MRI to sense the temperature
DNATURE.COM To comment online, click on Editorials at: go.nature.com/xhunqu inside the human body remotely. Now the chemists have reclaimed both the tool and the function. It is a proof of concept at this stage, but it does go some way towards opening that mysterious black box. 